\title{
A PAPR Reduction for OFDM Signals Based on Self-Adaptive Multipopulation DE algorithm
}

\author{
Hocine Ait-Saadi ${ }^{1}$, Jean-Yves Chouinard ${ }^{2}$, and Abderrazak Guessoum ${ }^{3}$ \\ ${ }^{1,3}$ Département de l'électronique,Université de Blida1, Algeria \\ ${ }^{2}$ Département de génie électrique et de génie informatique, Université Laval, Québec, Canada
}

\section{Article Info}

Article history:

Received: Mar 6, 2017

Revised: May 30, 2017

Accepted: Jun 16, 2017

\section{Keyword:}

OFDM

Peak-to-average power ratio

Partial transmit sequence

Differential evolution

algorithm.

\begin{abstract}
One of major drawbacks of orthogonal frequency division multiplexing (OFDM) systems is the high peak-to-average power ratio (PAPR). A signal with high PAPR leads to nonlinear distortion caused mainly by power amplifiers in wireless transmitters. Partial transmit sequence (PTS) is one of the most attractive methods to reduce the PAPR in OFDM systems. It achieves considerable PAPR reduction without distortion, but it requires an exhaustive search over all the combinations of the given phase factors, which results in a computational complexity that increases exponentially with the number of partitions. For this optimization problem, we propose in this paper a suboptimal PTS method based on the self-adaptive multipopulation differential evolution algorithm (SAMDE). The self adaptation of control parameters and structured population, is able to obtain high quality solutions with low computational cost by evolving each sub-population of individuals over successive generations.
\end{abstract}

\section{Corresponding Author:}

Hocine Ait-Saadi

Département de l'électronique

Université de Blida1, Route de Soumaa, BP 270, Blida Algeria

(+213) 25433850

Email: h_aitsaadi@univ-blida.dz

\section{INTRODUCTION}

Orthogonal frequency division multiplexing (OFDM) is widely used for high speed transmission technologies such as WIMAX, LTE, WIFI, DAB-T and DVB-T. The OFDM concept is based on spreading the high speed data to be transmitted over a large number of subcarriers. OFDM is useful and robust against multipath fading channels. However, the generation of OFDM signals typically induces large envelope fluctuations, known as Peak-to-Average Power Ratio (PAPR). The PAPR is defined as the ratio of the maximum instantaneous power and the average power of the signal to analyze. An OFDM signal with high PAPR transmitted through a nonlinear device, such as a high-power amplifier (HPA), leads to in-band or out-of-band signal distortion such as spectral regrowth, intermodulation, or constellation tilting and scattering [1], [2]. The Partial Transmit Sequences (PTS) method is one of the most attractive in reducing the PAPR for OFDM systems. It is a distortionless scheme for PAPR reduction in OFDM systems and it works with an arbitrary number of subcarriers and any modulation scheme. The principle of the PTS method is to partition the input data of $N$ symbols into $M$ disjoint subblocks [3]. The subcarriers in each subblock are weighted by a phase factor selected from a set of $W$ factors for that subblock. The phase factors are selected such that the PAPR of the combined signal is minimized. The conventional partial transmit sequence technique (C-PTS) has exponentially increased search complexity. Many methods have been proposed to reduce the number of candidate signals, which means decreasing the number of searches in the PTS scheme but with a compromise in PAPR reduction efficiency. Among them, there are iterative and simplified search methods such as, the flipping iterative method (IF-PTS) proposed in [4] or a gradient descent search (GD-PTS) proposed in [5].

Evolutionary algorithms have been also considered by many researchers for reducing the number of candidate signals in PTS scheme. Genetic algorithms are used for searching rotation factors while reducing the PAPR in PTS scheme (GA-PTS) [6]. The bee colony optimization algorithm (BCO-PTS) has been proposed in [7]. In this 
article, we propose a Self-Adaptive Multipopulation Differential Evolution (SAMDE) algorithm for searching the optimal phase factors vector required in the PTS scheme. The population in SAMDE algorithm is partitioned into small sub-populations known as islands. In this model, each sub-population is evolving independently from the others. The sub-populations exchange information between them in a process called migration. The dynamic adaptation of control parameters and the use of a structured population provide a way to increase the amount of potential search moves. Simulation results demonstrate that the performances of the SAMDE-PTS method are better in terms of PAPR reduction with lower numbers of candidate signals when compared to other optimization algorithms.

\section{OFDM SIGNAL AND PAPR}

In OFDM systems, the inverse fast Fourier transform (IFFT) is used to get the complex envelope in discrete time-domain $x_{n}$ which is given by:

$$
x_{n}=\frac{1}{\sqrt{N}} \sum_{k=0}^{N-1} X_{k} e^{j \frac{2 \pi k n}{L N}}, \quad n=0,1, \ldots, L N-1,
$$

where $N$ is the number of subcarriers, $L$ is the oversampling factor and $X=\left\{X_{k}, k=0, \ldots, N-1\right\}$ is a block of $N$ input symbols. Therefore, the PAPR of transmitted OFDM signal $x_{n}$ defined as the ratio of the maximum power to the average power, is expressed as

$$
\operatorname{PAPR}=\frac{\max _{0 \leq n \leq L N-1}\left|x_{n}\right|^{2}}{P_{a v}}
$$

where $P_{a v}=E\left[\left|x_{n}\right|^{2}\right]$ is the average power and $E[\cdot]$ denotes the expectation operation. The oversampling is done by inserting $(L-1) N$ zeros before IFFT module. The oversampling factor must be $(L \geq 4)$ for a good approximation of the PAPR of continuous-time OFDM signal [8].

The distribution of PAPR can be expressed in terms of complementary cumulative distribution function $(\mathrm{CCDF})$, which is also used to evaluate the performance of PAPR reduction in OFDM systems. It represents the probability $\zeta$ that the PAPR of an OFDM symbol exceeds a given threshold PAPR $_{0}$, which is denoted as

$$
\zeta=\operatorname{CCDF}\left(z_{0}=\operatorname{PAPR}_{0}\right)=\operatorname{Prob}\left\{\operatorname{PAPR}>z_{0}\right\}
$$

A relatively accurate approximation of the CCDF is proposed in [9], by employing the extreme value theory :

$$
\operatorname{CCDF}\left(z_{0}\right) \cong 1-\exp \left\{-N \mathrm{e}^{-z_{0}} \sqrt{\frac{\pi}{3} \ln N}\right\}
$$

Thus, for a given probability $\zeta$ and from (4) the threshold $z_{0}$ could be formulated as

$$
z_{0}=-\ln \left(\frac{\ln (1-\zeta)}{-N \sqrt{\frac{\pi}{3} \ln (N)}}\right)
$$

The Solid State Power Amplifier (SSPA) is an often used model of the nonlinear HPA. The SSPA produces no phase distortion and the AM/AM conversion function is given by

$$
A(|x(t)|)=\frac{\nu|x(t)|}{\left[1+\left(\nu|x(t)| / A_{0}\right)^{2 p}\right]^{1 / 2 p}}
$$

where $\nu$ is the amplification gain, $A_{0}=\nu A_{\text {sat }}, A_{\text {sat }}$ denotes the amplifier input saturation and $p$ determines the smoothness of the transition from the linear region to saturation region. The operating point of the SSPA in relationship with the nonlinearity of the HPA on a signal, depends on a quantity called the input back-off (IBO), defined as $I B O=10 \log _{10}\left(A_{\text {sat }}^{2} / E\left[|x(t)|^{2}\right]\right)$ in $\mathrm{dB}$. An OFDM signal with high PAPR, leads to nonlinear distortions which increase the BER. An HPA with high IBO has a large linear amplifier region with low distortion, but this leads to a poor power efficiency. An HPA with low back-off values increases nonlinear distortions as inter-modulation, in-band distortion and out-of-band radiation. These distortions result also in BER degradation. To prevent the occurrence of such problems, a suboptimal version of PTS technique reducing the PAPR of the transmitted signal, can be envisaged. 


\section{CONVENTIONAL PTS AND OPTIMIZATION PROBLEM}

In the conventional PTS (C-PTS) scheme, the input data block $X$ is evenly divided into $M$ disjoint subblocks, which are $X_{m}=\left[X_{m, 0}, X_{m, 1}, \ldots, X_{m, N-1}\right]^{T}$, such that

$$
X=\sum_{m=1}^{M} X_{m}
$$

with $m=1,2, \ldots, M$. The IFFT output of each subblock (i.e., $x_{m}=\left[x_{m, 0}, x_{m, 1}, \ldots, x_{m, L N-1}\right]^{T}$ is multiplied by a rotation factor $\left(b_{m}\right)$ selected from a $W$-element set, with $b_{m}=e^{j \phi_{m}}, m=1,2, \ldots, M$, and $\phi_{m} \in[02 \pi)$. For $W=2$, the allowed phase factors $b_{m}$ belong to the set $\{ \pm 1\}$, while for $W=4$ they belong to the $\{ \pm 1, \pm j\}$ set. Figure 1 shows the block diagram of a OFDM system using the conventional PTS technique. The PTS OFDM symbol is formed by adding the $M$ partitions as follows :

$$
\dot{x}_{n}(b)=\sum_{m=1}^{M} b_{m} \cdot x_{m, n}, n=0,1, \ldots, N L-1
$$

where $\dot{x}(b)=\left[\dot{x}_{0}(b), \dot{x}_{1}(b), \ldots, \dot{x}_{N L-1}(b)\right]^{T}$. Assuming that $W$ is the number of allowed phase factors, the determination of optimal vector with the phase rotation factors $b^{o p t}=\left[b_{1}, b_{2}, \ldots, b_{M}\right]^{T}$ minimizing the PAPR of the PTS OFDM signal, requires an exhaustive search over $C=W^{M-1}$ combinations.

$$
b_{\text {opt }}=\arg \min _{c=1}^{C} \frac{\max _{0 \leq n \leq L N-1}\left|\dot{x}_{n}(b)\right|^{2}}{P_{a v}}
$$

The optimal set of phase factors is sent to the receiver as a side information for correct decoding of the signal. It is

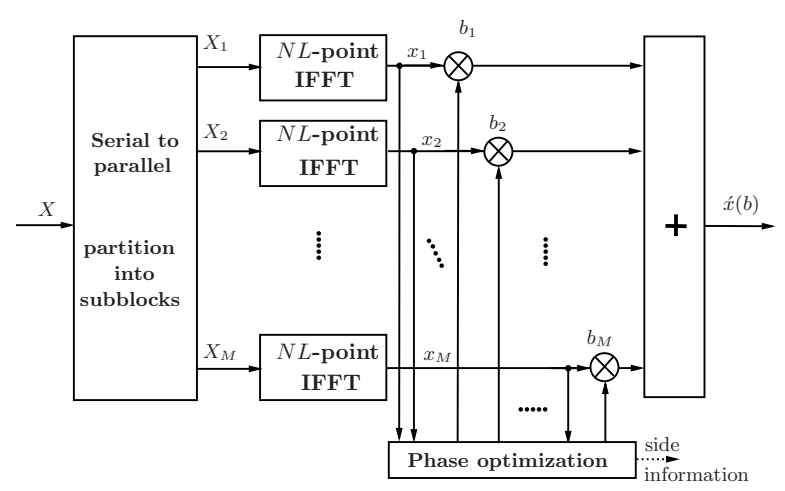

Figure 1. OFDM signal generation using the PTS technique.

possible to reduce the number of samples required for PAPR calculation by using a reduced complexity PTS scheme (RC-PTS) as proposed by [10]. Calculating the power of $x(b)$ in (8) and applying the Cauchy-Schwartz inequality

$$
\begin{aligned}
\left|\dot{x}_{n}(b)\right|^{2} & =\left|\sum_{m=1}^{M} b_{m} \cdot x_{m, n}\right|^{2} \\
& \leq \sum_{m=1}^{M}\left|b_{m}\right|^{2} \times \sum_{m=1}^{M}\left|x_{m, n}\right|^{2}=M Q_{n}
\end{aligned}
$$

where $Q_{n}=\sum_{m=1}^{M}\left|x_{m, n}\right|^{2}$ is the sum of power of the samples at time $n$ in the $M$ subblocks. Suppose that $\Phi_{\mathrm{N}}$ is the minimum possible peak power among the different time-domain symbols in an OFDM system with $N$ subcarriers, then we can write

$$
M Q_{n} \geq \max _{0 \leq n \leq L N-1}\left|\dot{x}_{n}(b)\right|^{2} \geq \Phi_{\mathrm{N}}
$$

and thus

$$
Q_{n} \geq \frac{\Phi_{\mathrm{N}}}{M}=\alpha
$$


Based on the above inequality and if $\Phi_{\mathrm{N}}$ is known, it is possible to consider only the samples with $Q_{n} \geq \alpha=\Phi_{\mathrm{N}} / M$ for PAPR calculation during the exhaustive search of optimal set of phase factors in PTS scheme. In practice it is not possible to get the true values of $\Phi_{\mathrm{N}}$ and $\alpha$, but an estimation of this threshold for selecting samples can be determined by using the CCDF function of the peak power similar to (4).

$$
\zeta=\operatorname{Prob}\left\{\max _{0 \leq n \leq L N-1}\left|\dot{x}_{n}(b)\right|^{2}>z_{0} \times P_{a v}\right\}
$$

where $\zeta$ is the probability that the peak power exceeds a given threshold $\Phi_{\mathrm{N}}^{\zeta}$; this threshold is obtained by using (5) as

$$
\Phi_{\mathrm{N}}^{\zeta}=z_{0} \times P_{a v}=-P_{a v} \times \ln \left(\frac{\ln (1-\zeta)}{-N \sqrt{\frac{\pi}{3} \ln (N)}}\right)
$$

The probability of event $Q_{n}>\alpha$ is expressed as [10],

$$
\begin{array}{r}
p_{\alpha}=\left(\frac{M}{P_{a v}}\right)^{M-1} \frac{e^{\frac{-\alpha M}{P_{a v}}}}{\Gamma(M)}\left[\alpha^{M-1}+\frac{P_{a v}}{M}(M-1) \alpha^{M-2}+\left(\frac{P_{a v}}{M}\right)^{2}(M-1)(M-2) \alpha^{M-3}+\cdots\right. \\
\left.+\left(\frac{P_{a v}}{M}\right)^{M-1}(M-1) !\right]
\end{array}
$$

where $\Gamma(\cdot)$ is the gamma function. In RC-PTS scheme, the average number of samples used for PAPR calculation is then given by $p_{\alpha} \times L \times N$ for each candidate signal, and therefore the computational complexity is reduced. The complexity reduction is more significant for small values of $M$ (i.e. $M=4$ ).

\section{PROPOSED PTS SCHEME BASED ON DIFFERENTIAL EVOLUTION ALGORITHM}

In this section, we describe the self-adaptive multipopulation differential evolution algorithm (SAMDE). This algorithm, based on a multiple populations structure and self adaptation of control parameters, is used to search the optimal phase rotation factors vector.

\subsection{Differential evolution algorithm}

The differential evolution algorithm (DE) is a population based search approach introduced by [11] and considered as an improved version of the original genetic algorithm introduced by [12]. The DE involves several control parameters such as mutation weighting factor $F$, crossover control parameter $C R$, population size $N P$ and fitness function to minimize $f$. A population $\mathcal{P}$ consists of $N P$ parameter vectors $\Theta_{i, G},(i=1,2, \ldots, N P$ for each generation $G$ ) and each vector is called an individual. The initial population is chosen randomly and should cover the entire parameter space, whereas the new individuals are generated by using the following operators:

1. Mutation is an important operator which consists in adding a perturbation on the population. Basically during each generation $G$, mutant individuals $v_{i, G+1}$ are produced by adding the weighted difference between two or more population vectors, resulting in a third vector. There are several variant strategies of DE [13]:

$$
\begin{aligned}
\text { DE/rand } / 2: & v_{i, G+1}=\Theta_{r_{1}, G}+F\left(\Theta_{r_{2}, G}-\Theta_{r_{3}, G}+\Theta_{r_{4}, G}-\Theta_{r_{5}, G}\right) \\
\text { DE/best/1 : } & v_{i, G+1}=\Theta_{\text {best }, G}+F\left(\Theta_{r_{1}, G}-\Theta_{r_{2}, G}\right) \\
\text { DE/best/2 : } & v_{i, G+1}=\Theta_{\text {best }, G}+F\left(\Theta_{r_{1}, G}-\Theta_{r_{2}, G}+\Theta_{r_{3}, G}-\Theta_{r_{4}, G}\right) \\
\text { DE/rand - to - best/1 : } & v_{i, G+1}=\Theta_{i, G}+F\left(\Theta_{\text {best }, G}-\Theta_{i, G}\right)+F\left(\Theta_{r_{1}, G}-\Theta_{r_{2}, G}\right) \\
\text { DE/rand - to - best/2 }: & v_{i, G+1}=\Theta_{i, G}+F\left(\Theta_{b e s t, G}-\Theta_{i, G}\right)+ \\
& F\left(\Theta_{r_{1}, G}-\Theta_{r_{2}, G}+\Theta_{r_{3}, G}-\Theta_{r_{4}, G}\right)
\end{aligned}
$$

where $r_{1} \neq r_{2} \neq r_{3} \neq r_{4} \neq r_{5} \neq i(\in\{1,2, \ldots, N P\})$ are random indexes and $\Theta_{\text {best }, G}$ is the best individual in the population at current generation $G$. Parameter $F \in[0,1]$ controls the amplification of the difference vector of the randomly chosen individuals.

2. Crossover is a genetic operator designed to increase the diversity of the population using the following scheme:

$$
u_{i, G+1}= \begin{cases}v_{i, G+1} & \text { if } r_{[0,1]} \leq C R \\ \Theta_{i, G} & \text { if } r_{[0,1]}>C R\end{cases}
$$


where $C R(C R \in[0,1])$ is the crossover probability or crossover control parameter and $r$ denotes a random number. The new trial individuals $u_{i, G+1}$ of the next generation are produced by exchanging individuals from the previous generation population $\Theta_{i, G}$ with the mutated vectors $v_{i, G+1}$.

3. Selection is a DE operator applied to select the fittest individuals of the resulting offspring $u_{i, G+1}$ for the next generation according to their fitness scores $f$ as expressed by:

$$
\Theta_{i, G+1}= \begin{cases}u_{i, G+1} & \text { if } f\left(u_{i, G+1}\right)<f\left(\Theta_{i, G}\right) \\ \Theta_{i, G} & \text { otherwise }\end{cases}
$$

The control parameters of the DE algorithm $F, C R$ and $N P$ are generally fixed during the whole optimization process. But with fixed values, after a number of generations, the search is performed mainly in the neighborhood of the promising solutions, this reduces the exploration of the whole search space and involves a stagnation effect.

\subsection{Self-Adaptive Multipopulation DE algorithm}

The main drawback of the classical DE algorithm after a number of generations is its limited capability to produce new promising solutions by exploring correctly the decision space. Therefore, the optimization process requires more search moves to find the optimal or the suboptimal solution, which is not suitable for our objectives. To overcome the problem of stagnation and dynamically adjust the control parameters $F$ and $C R$, we adopt two approaches. The first one, is to use a self-adaptive version of DE algorithm (SADE) developed by [14]; the control parameters are determined in accordance with the evaluation of the uniform random numbers:

$$
\begin{aligned}
F_{i, G+1} & = \begin{cases}F_{\text {low }}+F_{\text {upp }} \times \text { rand }_{1} & \text { if } \text { rand }_{2}<\tau_{1} \\
F_{i, G} & \text { otherwise }\end{cases} \\
C R_{i, G+1} & = \begin{cases}\operatorname{rand}_{3} & \text { if } \text { rand }_{4}<\tau_{2} \\
C R_{i, G} & \text { otherwise }\end{cases}
\end{aligned}
$$

where $\tau_{1}, \tau_{2}$ denote the probabilities to adjust the control parameters $F$ and $C R$. The numbers $\operatorname{rand}_{1}$ to $\operatorname{rand}_{4}$ are random values in the interval $[0,1]$. For the values $F_{\text {low }}=0.1$ and $F_{\text {upp }}=0.9$, the new parameters $F$ and $C R$ are randomly generated within intervals $[0.1,1]$ and $[0,1]$, respectively. The updates are obtained before the mutation is performed. The objective is to reach the best solution with a minimum number of searches. The second one, to increase the population diversity and to enhance the space search exploration, is to use a structured population or multipopulation structure. The space problem is subdivided into separated optimized sub-spaces. Many variants of the DE algorithm with a structured populations and different topologies have been proposed in literature [15], [16].

\subsection{Suboptimal search of PTS phase factors based on the SAMDE Algorithm}

In this section, a detailed description of the SAMDE algorithm used for searching the nearly optimal phase factors vector for PAPR reduction with PTS scheme (SAMDE-PTS), is examined. The phase factors search can be considered as a combinatorial optimization problem. The objective is to find out the best weighting factors vector $b$ that minimizes the PAPR function. The fitness function is directly related to the PAPR and is defined as:

$$
\begin{aligned}
& f(b)=\frac{\max \left[\left|\hat{x}_{n}(b)\right|^{2}\right]}{E\left[\left|\hat{x}_{n}(b)\right|^{2}\right]} \text { with } 0 \leq n \leq L N-1 \\
& \text { subject to } b \in\left\{e^{j \phi_{m}}\right\}^{M} \text { where } \phi_{m} \in\left\{\frac{2 \pi k}{W} \mid k=0,1, \ldots, W-1\right\}
\end{aligned}
$$

To reduce the samples required for PAPR calculation at this step, R-PTS scheme is used. A given probability $\zeta$ to find the peak, and a threshold is deduced by using (14). This threshold is used to find the sample required for PAPR calculation by using (12), only an average of $p_{\alpha} L N$ samples are needed instead of $L N$ samples. The first step of SAMDE-PTS algorithm is to generate randomly an initial population $\mathcal{P}$ of $N P$ vectors or individuals $\Theta_{i, G}$ and each vector contains $M$ real phases randomly initialized with $\phi_{\text {im }} \in[0,2 \pi)$. In this work, the population $\mathcal{P}$ is structured in $S$ sub-populations of $n_{p}$ individuals. Each sub-population $\mathcal{P}_{j}(j \in\{1,2, \ldots, S\})$, evolves independently towards a solution as per the self-adaptive DE algorithm. The population generated contains phases with real values, but the phase factors $b_{i, m}$ required for fitness evaluation in (25) being in discrete form, we need to transform (or to map) each new phase into the set of discrete allowed phases $\left\{\phi_{m}\right\}$ and determine the corresponding phase factors $\left\{b_{i, m}\right\}$. We 


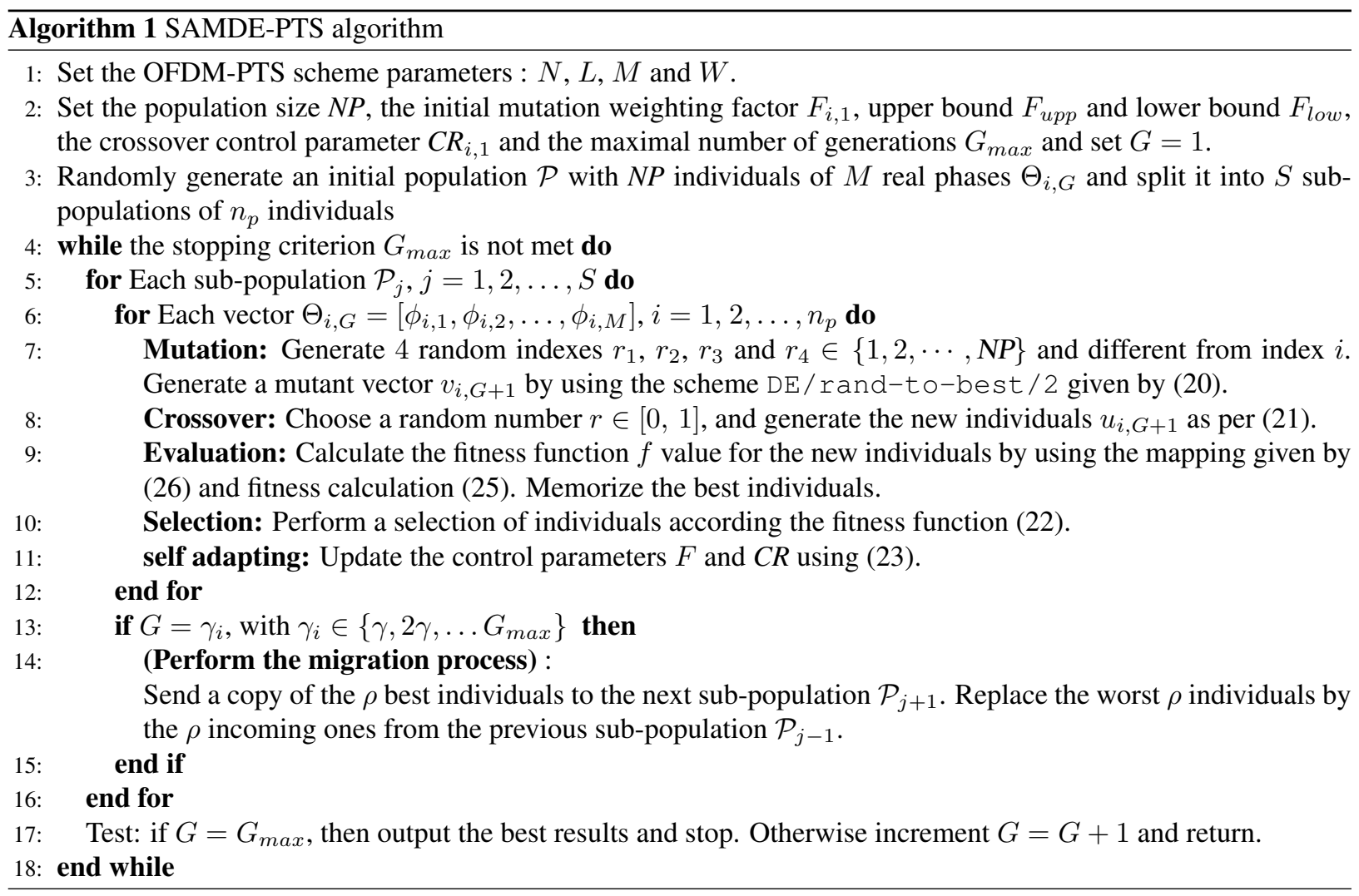

consider the case where $W=4$ and the allowed phases factors are $\{+1,+j,-1,-j\}$. This mapping operation is performed only for evaluating the objective function, without overwriting the populations and expressed as:

$$
b_{i, m}=\left\{\begin{array}{cl}
1 & \text { if } 7 \pi / 4 \leq \phi_{i, m}<\pi / 4 \\
j & \text { if } \pi / 4 \leq \phi_{i, m}<3 \pi / 4 \\
-1 & \text { if } 3 \pi / 4 \leq \phi_{i, m}<5 \pi / 4 \\
-j & \text { if } 5 \pi / 4 \leq \phi_{i, m}<7 \pi / 4
\end{array}\right.
$$

During one generation, for each vector of each sub-population, a self-adaptive DE is employed with mutation, crossover and selection operations to produce an offspring and to select one of these vectors with the best fitness value. Updating the sub-populations independently with a migration process ensures a better exploration of the decision space. The mutation adopted for each sub-population is the DE/rand-to-best/2 scheme. Initially the control parameters are randomly generated for each sub-population and updated in each generation using (23 and 24). The multiple populations structure decreases the risk of stagnation which might occurs with the DE algorithm after a number of generations. For each sub-population $\mathcal{P}_{j}$, a migration mechanism is performed every $\gamma$ generations, by sending a copy of the $\rho$ best individuals to the next sub-population, where $\gamma \in \mathbb{N}$ is called the migration interval and $\rho \in \mathbb{N}$ represents the migration rate defined as the number of individuals which migrate between sub-populations. At the same time, each sub-population receives the $\rho$ best individuals from the previous sub-population which will replace the same number of the worst individuals, even if they are not better. This mechanism adds new search moves and enhances the algorithm performance. The proposed SAMDE algorithm can be summarized in Algorithm 1 and a flow chart is given by Figure 2 with 4 sub-populations and unidirectional topology ring.

\subsection{Complexity analysis}

The C-PTS method requires a high complexity search by trying $C=W^{M-1}$ candidate signals to find the optimum set of phase factors. The computational complexity is $(L N M C+L N C)$ complex multiplications and $(2 L N C(M-1)+L N C-1)$ real additions. The amount of PAPR reduction increases with the number of subblocks $M$ and the number of allowed phase factors $W$, but at the cost of high computational complexity. For R-PTS scheme [10], the average number of samples required for PAPR calculation is reduced to $p_{\alpha} L N$, such that the complexity is around $\left(L N M+p_{\alpha}(L N M+L N) C\right)$ complex multiplications and $\left(3 L N M+p_{\alpha}(2 L N M-L N) C-2 L N\right)$ 


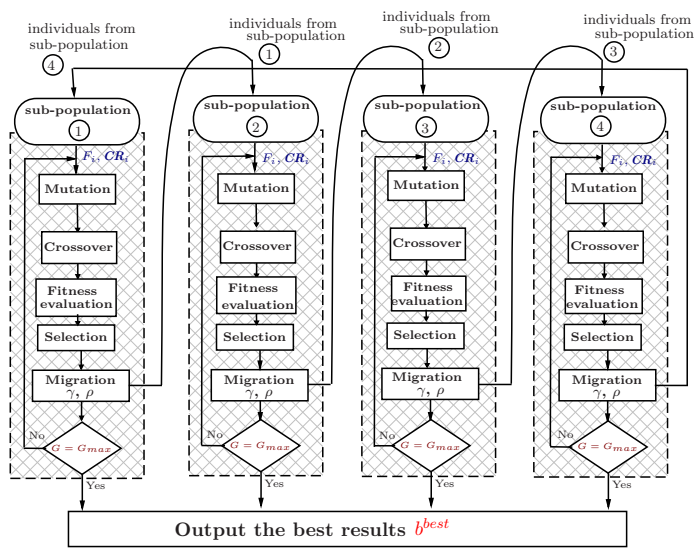

Figure 2. Flow chart of multipopulation DE algorithm with 4 sub-populations and unidirectional ring topology.

real additions. For all suboptimal PTS methods trying to reduce the candidate signals, the computational complexity is proportional to the number of phase factors searches. For heuristic methods, such as the gradient descent search (GD-PTS) algorithm used in [5], the number of searches is given by $C_{(M-1)}^{r} W^{r} \times I$, where $r$ is the radius of the neighborhood, $I$ is the number of iterations and $C_{n}^{k}$ is the binomial coefficient. With the iterative flipping algorithm for PTS (IF-PTS) [4], the search complexity is proportional to $(M-1) \times W$. For stochastic methods based on population search as the proposed method (SAMDE-PTS), the artificial bee colony algorithm (ABC-PTS) [7], the differential evolution algorithm (DE-PTS) considered in [17],[18] and the genetic algorithm (GA-PTS) [6], a PAPR calculation is needed at each iteration for each candidate, so the number of searches is given by the population size times the number of iterations (cycles $C$ or generations $G$ ) $N P \times I$. The computational complexity of the proposed scheme is also evaluated by using the CCRR (computational complexity reduction ratio) defined as follows:

$$
C C R R=\left(1-\frac{\text { complexity of proposed PTS }}{\text { complexity of C-PTS }}\right) \times 100 \%
$$

\section{SIMULATION RESULTS}

Extensive simulations have been conducted to verify the performance of the SAMDE-PTS scheme for searching the optimal combination of phase factors. An OFDM system with 16-QAM modulation and $N=1024$ subcarriers with an oversampling rate of $L=4$ is simulated. To generate the CCDF of PAPR, $10^{4}$ random OFDM symbols are used. For PAPR reduction technique with PTS scheme, the allowed rotation phase factors are described in section 3 .
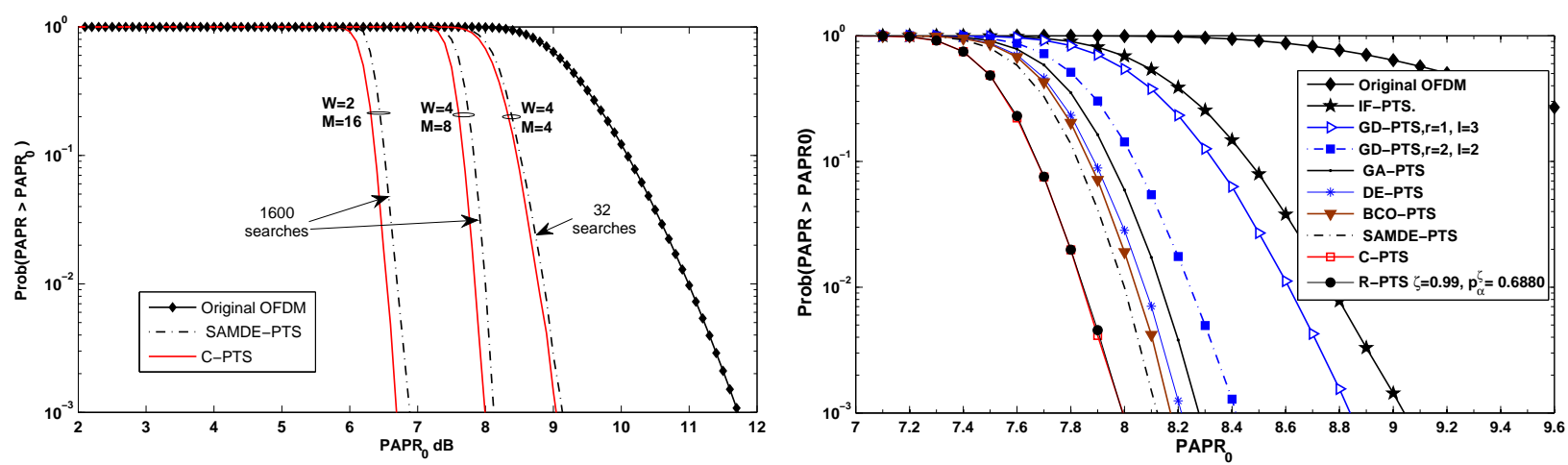

Figure 3. CCDF's of original OFDM, conventional PTS Figure 4. CCDF's comparison of PAPR reduction with and the proposed SAMDE-PTS method conventional PTS, SAMDE-PTS and some other heuristic and meta-heuristic methods

Figure 3 shows the CCDF curves of the original OFDM signal (without any PAPR reduction method), the 
Table 1. Search cost of the different methods and the PAPR values when $\mathbf{C C D F}=10^{-3}$.

\begin{tabular}{|l|l|c|c|}
\hline Method & Number of searches & CCRR $\%$ & PAPR dB \\
\hline C-PTS & $C=W^{M-1}=16384$ & 0 & 8.00 \\
\hline R-PTS $\left(\zeta=0.99, p_{\alpha}^{\zeta}=0.68\right)[10]$ & $C=16384$ & 31.1 & 8.0 \\
\hline R-PTS $\left(\zeta=0.4, p_{\alpha}^{\zeta}=0.3735\right)[10]$ & $C=16384$ & 62.64 & 8.0 \\
\hline SAMDE-PTS $\left(\zeta=0.99, p_{\alpha}^{\zeta}=0.68\right)$ & $S \times n_{p} \times G=1600$ & 93.27 & 8.137 \\
\hline SAMDE-PTS $\left(\zeta=0.4, p_{\alpha}^{\zeta}=0.3735\right)$ & $S \times n_{p} \times G=1600$ & 96.34 & 8.156 \\
\hline SAMDE-PTS & $S \times n_{p} \times G=1600$ & 90.23 & 8.12 \\
\hline BCO-PTS [7] & $N P \times C=1600$ & 90.23 & 8.17 \\
\hline DE-PTS $[17,18]$ & $N P \times G=1600$ & 90.23 & 8.20 \\
\hline GA-PTS $[6]$ & $N P \times G=1600$ & 90.23 & 8.27 \\
\hline GD-PTS $(r=2, I=2)[5]$ & $C_{7}^{2} W^{2} \times 2=672$ & 95.89 & 8.41 \\
\hline GD-PTS $(r=1, I=3)[5]$ & $C_{7}^{1} W^{1} \times 3=84$ & 99.48 & 8.84 \\
\hline IF-PTS $[4]$ & $(M-1) \times W=28$ & 99.82 & 9.35 \\
\hline OFDM only & 0 & - & 11.7 \\
\hline
\end{tabular}

PAPR reduction is achieved by using the conventional PTS (C-PTS) and the proposed SAMDE-PTS method. The CPTS method requires $C=W^{M-1}$ candidate signals. This corresponds to 64,16384 and 32768 searches for $(W, M)$ given by $(4,4),(4,8)$ and $(2,16)$, respectively. The PAPR reduction is enhanced by increasing $M$, but at the expense of an exponential increase in the search computational complexity. For the proposed SAMDE-PTS method and for ( $M=8$ or $\left.16, S=4, n_{p}=10, N P=40, \rho=1, \gamma=10\right)$, different values of $F$ and $C R$ are initially assigned to each subpopulation after a few generations, self adaptation is performed with a search cost of 1600 . Whereas, for ( $M=4, S=2, n p=4, G=4)$ the total search cost is 32 . For almost the same PAPR reduction performance, the computational complexity has been considerably reduced with CCRR's $=95.11 \%, 90.24 \%$ and $50 \%$ for $M=16,8$ and 2 respectively.

Figure 4 shows the different CCDF simulated curves of the PAPR with a variety of heuristic and metaheuristic methods. Table 1 gives the PAPR at CCDF $=10^{-3}$ and lists the search costs for $(N=1024, L=4$, $W=4, M=16$ ). The IF-PTS method with only 28 searches and CCCR=99.829\%, presents the lower computational complexity but with considerably the worst performance in PAPR reduction. The meta-heuristic methods give a better performance in PAPR reduction with the same search complexity, which corresponds to CCRR $=90.24 \%$. But, the best performance is achieved by the proposed SAMDE-PTS method with a PAPR equal to $8.125 \mathrm{~dB}$. It can be seen that the proposed method outperforms all the other methods in PAPR reduction while keeping a low complexity of 1600 .

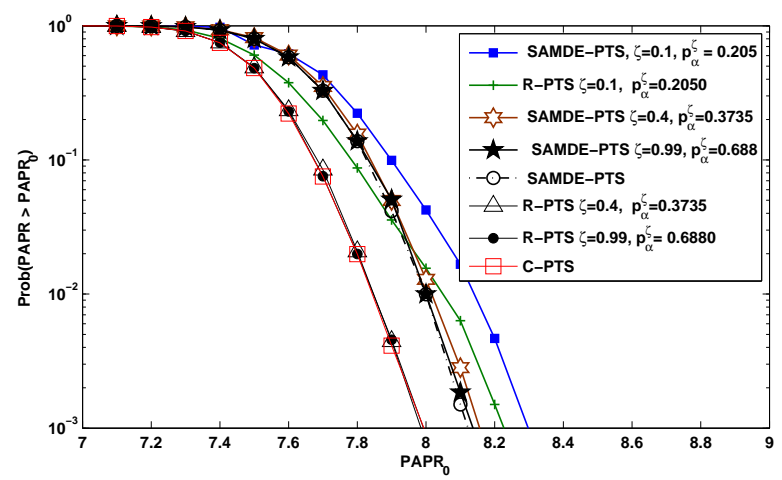

Figure 5. Comparison of PAPR reduction performance for the C-PTS, R-PTS and the proposed method SAMDE-PTS based on R-PTS scheme with $W=4, M=8$.

Figure 5 shows the performance of the conventional PTS and R-PTS scheme with exhaustive search over 16384 candidate signals, and the proposed method SAMDE-PTS with 1600 searches. For the PAPR calculation required for each generation in optimization process, the simulations are done by taking all $L N$ samples, and also by taking about $p_{\alpha}^{\zeta} L N$ samples and with different values of $\zeta$. The proposed PTS scheme with $(\zeta \geq 0.40)$ can achieve 
almost the same PAPR reduction performance but with lower computational complexity reaching a CCRR of $96.34 \%$.

Figure 6 depicts the BER versus $E_{b} / N_{0}$ performance of OFDM signals over the AWGN channel when SSPA has been considered with different parameters $p=2$ and 3 and operating at IBO $=3$ and $6 \mathrm{~dB}$. The best performance bound curve is obtained with no SSPA; the nonlinearity effects of SSPA are neglected. The SSPA with low input back-off value $(I B O=3 \mathrm{~dB})$ increases the BER of the system. The small IBO values (IBO $=0,3,6 \mathrm{~dB})$ lead to inband distortion that increases the BER of the system. Parameter $p$ controls the AM/AM sharpness of the saturation region and affects the BER performance. The proposed SAMDE-PTS scheme with $\zeta=0.4, p_{\alpha}^{\zeta}=0.3735$ offer an improved BER performances over AWGN channel compared with the original OFDM system with SSPA, and almost the same BER performance as that obtained with the C-PTS scheme.

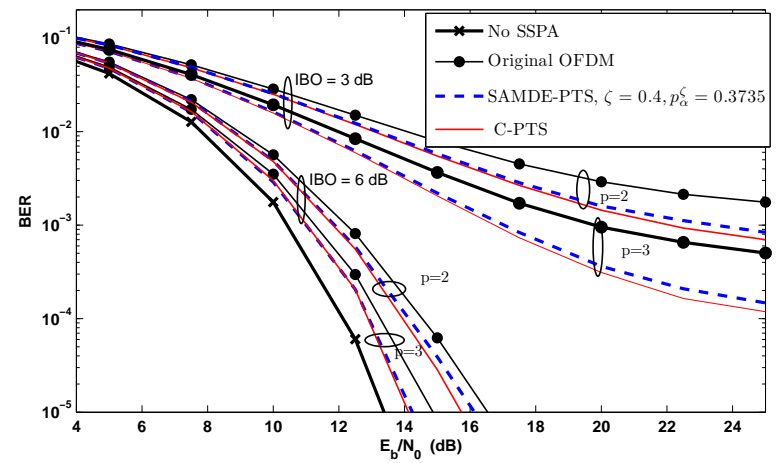

Figure 6. BER vs $E_{b} / N_{0}$ performance of SAMDE-PTS and C-PTS methods with $W=4 M=8$, over an AWGN channel and by using an SSPA $(p=2$ and $3, \mathrm{IBO}=3$ and $6 \mathrm{~dB})$.

\section{CONCLUSION}

In this paper, we have proposed a new approach to reduce the PAPR in OFDM systems by using a SelfAdaptive Multipopulation Differential Evolution Partial Transmit Sequence algorithm (SAMDE-PTS) for searching the optimal combination of phase factors in PTS technique. Simulation results have shown that the proposed method achieves almost the same PAPR reduction and the same BER performance as that of the conventional PTS scheme while significantly reducing computational complexity by 10 . Furthermore, simulation results have shown that SAMDE-PTS method outperforms others heuristic and meta-heuristic methods. In fact, the performance of the algorithm is enhanced by adopting a dynamic adaptation of control parameters and a multipopulation structure. This approach accelerates convergence and avoids stagnation by adding a new search moves and maintaining the population diversity.

\section{REFERENCES}

[1] E. Costa, M. Midrio, and S. Pupolin, "Impact of Amplifier Non-linearities on OFDM Transmission System Performance," Commun. Letters, vol. 3, no. 2, Feb. 1999.

[2] S. P. Yadav and S. C. Bera, "PAPR Reduction for Improved Efficiency of OFDM Modulation for Next Generation Communication Systems ," International Journal of Electrical and Computer Engineering (IJECE), vol. 6, no. 16, pp. $2310-2321,2016$.

[3] Z. T. Ibraheem, M. M. Rahman, S. N. Yaakob, M. S. Razalli, F. Salman, and K. K. Ahmed, "PTS Method with Combined Partitioning Schemes for Improved PAPR Reduction in OFDM System ," Indonesian Journal of Electrical Engineering and Computer Science (IJEECS), vol. 12, no. 11, pp. 7845 - 7853, Nov 2014.

[4] L. J. Cimini and N. R. Sollenberger, "Peak-to-Average Power Ratio Reduction of an OFDM Signal Using Partial Transmit Sequences," Commun. Letters, vol. 4, no. 3, Mar. 2000.

[5] S. H. Han and J. H. Lee, "PAPR Reduction of OFDM Signals Using a Reduced Complexity PTS Technique," IEEE Signal Processing Letters, vol. 11, no. 11, pp. 887-890, Nov. 2004.

[6] H. Liang, Y.-R. Chen, Y.-F. Huang, and C.-H. Cheng, "A modified genetic algorithm PTS technique for PAPR reduction in OFDM systems," in 15th Asia-Pacific Conf. on commun. APCC, Oct. 2009, pp. 182-185.

[7] Y. Wang, W. Chen, and C. Tellambura, "A PAPR reduction method based on artificial bee colony algorithm for OFDM signals,” IEEE Trans. Wireless Comm., vol. 9, pp. 2994-2999, Oct. 2010. 
[8] J. Tellado, "Peak to Average Power Reduction for Multicarrier Modulation," PhD thesis, Stanford University, Sep. 1999.

[9] S. Q. Wei, D. L. Goeckel, and P. E. Kelly, "A modem extreme value theory approach to calculating the distribution of the peak-to-average power ratio in OFDM systems," IEEE Inter. Conf. on Comm., vol. 3, pp. 1686-1690, Apr. 2002.

[10] S.-J. Ku, C.-L. Wang, and C.-H. Chen, "A reduced-complexity PTS-based PAPR reduction scheme for OFDM systems,” IEEE Trans. Wireless Comm., vol. 9, pp. 2455-2460, Aug. 2010.

[11] R. Storn and K. Price, "Differential Evolution-A Simple and Efficient Heuristic for global Optimization over Continuous Spaces," Springer, Journal of Global Optimization, vol. 11, no. 4, pp. 341-359, Dec. 1997.

[12] J. H. Holland, Adaption in Natural and Artificial Systems. Cambridge, MA: MIT Press, 1975.

[13] K. Price, R. Storn, and J. Lampinen, Differential Evolution: A Practical Approach to Global Optimization, ser. Natural Computing Series. Springer, 2005.

[14] J. Brest, S. Greiner, B. Boskovic, M. Mernik, and V. Zumer, "Self-adapting control parameters in differential evolution: A comparative study on numerical benchmark problems," IEEE Trans. on Evol. Comp., vol. 10, no. 6, pp. $646-657$, Dec. 2006.

[15] E. Alba and M. Tomassini, "Parallelism and evolutionary algorithms," IEEE Trans. on Evolutionary Computation, , vol. 6, no. 5, pp. 443 - 462, Jan. 2002.

[16] M. Weber, F. Neri, and V. Tirronen, "A study on scale factor in distributed differential evolution," Information Sciences, vol. 181, no. 12, pp. 2488-2511, 2011.

[17] H. Ait-Saadi, A. Guessoum, and J.-Y. Chouinard, "Differential evolution algorithm for PAPR reduction in OFDM systems," in 7th WOSSPA, May 2011, pp. $175-178$.

[18] H. Ait-Saadi, J.-Y. Chouinard, and A. Guessoum, "Distributed differential evolution algorithm for PAPR reduction of OFDM signals," in Intern. Conf. on Inf. Science, Signal Process. and their Applic., 2012, pp. 567-572.

\section{BIOGRAPHY OF AUTHORS}

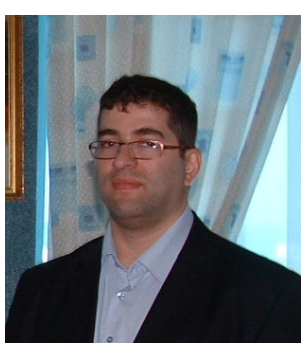

Hocine AIT-SAADI is a lecturer with the department of Electronics, Université de BLIDA. His research interests are focused on digital signal processing, Bayesian estimation, digital communication, multicarrier systems, wireless communication systems, metaheuristic algorithms. has received a BS degree in electrical engineering from the Université de BLIDA, Algeria, in 1998 and M.Sc Degree in 2001. He then attended Institut National Polytechnique de la lorraine in Nancy, France, where he obtained, a M.Sc in Communications, Control \& signal processing in 2002. In 2010 he was a visiting researcher at Laval University LRTS laboratory.

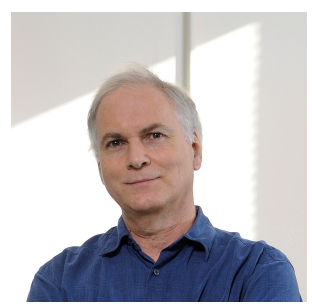

Jean-Yves Chouinard is a Professor with the Department of Electrical and Computer Engineering at Université Laval, Quebec city, Canada. His research interests are wireless communications, secure communication networks and signal processing for radar applications. He is the author/coauthor of more than 200 journal, conference papers and technical reports. He was co-recipient of the 1999 Neal Shepherd Best Propagation Paper Award from the IEEE Vehicular Society and of the 2004 Signal Processing Best Paper Award from the European Journal of Signal Processing. He is an editor of a book on information theory and co-author of book chapters on MIMO wireless communication systems and on OFDM-based mobile broadcasting. He is an Editor for the IEEE Transactions on Vehicular Technology and Associate Editor for the IEEE Transactions on Broadcasting.

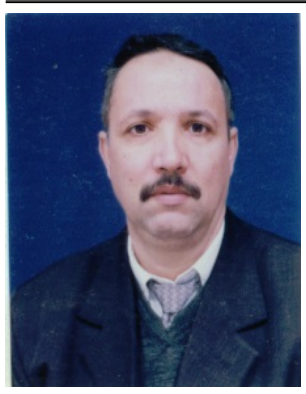

Abderrezak GUESSOUM has received a BS degree in electronics from the Ecole Nationale Polytechnique d'Alger, Algeria, in 1976. He then attented Georgia Institute of Technology in Atlanta, $\mathrm{Ga}$, USA, where he obtained, a PhD in electrical engineering, in 1984. He worked as an Assistant Professor in the School of Computer Science at Jackson State University, Mississippi, USA, from 1984 to 1985 . He has been with the Université de Blida, Algeria, as a Professor in the department of Electronics, since 1988. He is the head of the Digital Signal and Image Processing Research Laboratory. His current research interests include embedded signal processing algorithms, digital filter design, intelligent control, neural networks, genetic algorithms, and fuzzy logic. 